\title{
Extended ionized-gas structures in Seyfert 2 galaxy Mrk 78
}

\author{
D.V. Kozlova ${ }^{1,2}$, A.V. Moiseev ${ }^{2}$ and A.A. Smirnova ${ }^{2}$ \\ 1 Laboratory of Astrochemistry and Extraterrestial Physics, Ural Federal \\ University, 620002, Russia \\ 2 Special Astrophysical Observatory of the Russian Academy of Sciences, \\ 369167, Russia
}

Received: July 31, 2019; Accepted: October 24, 2019

\begin{abstract}
The search and study of extended emission-line regions (EELRs) related to AGN in early-type galaxies is interesting to probe the history of nuclear ionization activity and also to understand the process of external gas accretion. In this work, we present observations of the EELR in Mrk 78 obtained at the 6-m Russian telescope using the long-slit and 3D spectroscopy methods. We show that ionized-gas clouds at $12-16 \mathrm{kpc}$ projected distances from the nucleus are ionized by the AGN radiation. Also we have checked if the galaxy appearing in the optical images in the immediate neighbourhood of Mrk 78 near the external clouds is a dwarf companion or a part of a tidal structure. However, the spectrum of this galaxy, SDSS J074240.37+651021.4, obtained at the $6-\mathrm{m}$ telescope corresponds to a distant background galaxy with $z=0.38$.
\end{abstract}

Key words: galaxies: individual: Mrk 78 - galaxies: active - galaxies: Seyfert - galaxies: interactions - galaxies: ISM

\section{Introduction}

According to the unified model of active galactic nuclei (AGN), the radiation of the central accretion engine comes out in broad axisymmetrical cones collimated by the circumnuclear obscuring torus. The impact of AGN ionizing radiation together with radio jet mechanical energy on the surrounding interstellar medium appears in the shape of ionizing cones from hundreds pc to a few kpc in typical size. In some fraction of Seyfert galaxies, extended emission-line regions (EELRs) are also detected at radial distances of tens of kpc. EELRs can be used to measure the AGN energetic output at both time and spatial scales and also to study the gas distribution in the galactic environment. Indeed, a significant faction of EELR traces the off-plane gas related with tidal debris (Keel et al., 2012 ) or even the gas in a companion galaxy (Keel et al., 2019). One of the intriguing cases is the well-known AGN Mrk 6: deep imaging and spectroscopic observations revealed an extended system of gaseous filaments up to $40 \mathrm{kpc}$ from the nucleus accreted by the Seyfert galaxy (Smirnova et al., 2018). Therefore, 
the detailed study of EELRs is topical even around well-studied nearby active galaxies.

The Seyfert 2 galaxy Mrk 78 is a good illustration of the picture described above. The spectrophotometric properties of the inner $\left(r<4-5^{\prime \prime}\right.$, or $\left.3-4 \mathrm{kpc}\right)$ elongated emission-line structure studied with HST (Whittle \& Wilson, 2004; Kewley et al., 2006) can be explained in terms of jet-cloud interaction together with the mass outflow and a cone of UV-radiation emerging from the Seyfert nucleus (Fischer et al., 2011, see the corresponding HST image in Fig. 1). Groundbased narrow-band images obtained by De Robertis (1987) and Pedlar et al. (1989) also revealed a weak extended ionized gas emission outside the central region with an extensive asymmetric structure at $r>15^{\prime \prime}$ in the south-west. The presence of the gas emitting in [O III] in the western direction up to 12-14" was confirmed in spectroscopic observations by Unger et al. (1987). Also, Afanasiev \& Silchenko (1991) detected the [O III] line emission even at $r=18-20^{\prime \prime}$. Pedlar et al. (1989) have shown that the weak narrow-line region extending out to $10 \mathrm{kpc}$ to the west is consistent with AGN ionizing cones, however, the actual ionization state of the most distant part of the EELR is still unknown. Mrk 78 was included in the sample of the confirmed EELR selected from the Galaxy Zoo survey, but the follow-up spectra were obtained along $P A=90^{\circ}$ (Keel et al., 2012 ), therefore, the properties of the southwestern external emission knots were not measured.

In this paper, we analysed the large-scale ionized gas distribution and spectral properties of the most distant parts of the EELR in Mrk 78 derived from new long-slit and 3D spectroscopic observations at the 6-m telescope of Special Astrophysical Observatory of Russian Academy of Sciences (SAO RAS). The gas and stellar kinematics will be considered in the forthcoming paper. In the present paper, we assumed a distance to the galaxy of $165 \mathrm{Mpc}$ with a corresponding scale of $0.80 \mathrm{kpc} /{ }^{\prime \prime}$ (according to $\mathrm{NED}^{1}$ ).

\section{Observations and data reduction}

Observations were carried out at the prime focus of the 6-m SAO RAS telescope with the multi-mode focal reducers: SCORPIO (Afanasiev \& Moiseev, 2005) and SCORPIO-2 (Afanasiev \& Moiseev, 2011). We used the 3D spectroscopy with the Fabry-Perot interferometer (FPI), long-slit spectroscopy, and direct-image modes. Also, we used the data obtained with the integral-field Multi-Pupil Field Spectrograph (Afanasiev et al., 2001). The log of observations and parameters of the used instruments are listed in the Table 1.

SCORPIO and SCORPIO-2 both have the 6.1 fields of view with the 0.36 arcsec pixel size. The slit has the 6.1 length and the $1^{\prime \prime}$ width. The scanning FPI mapped the spectral region around the redshifted [O III] $\lambda 4959$ emission line. We made the binning of these data to reach a higher signal-to-noise ratio

${ }^{1}$ http://ned.ipac.caltech.edu/ 

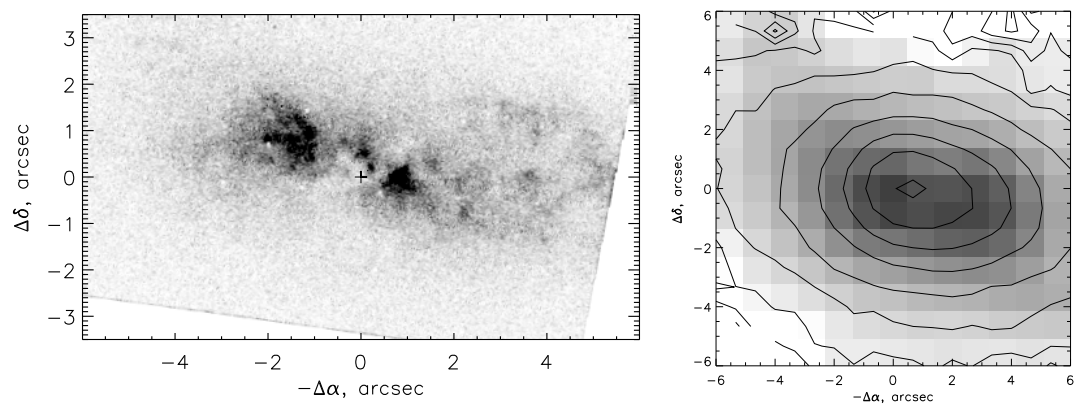

Figure 1. Left: the HST/FOC image with the F520M filter showing the [O III] emission (see Fischer et al., 2011). Right: the MPFS image in the [O III] $\lambda 5007$ line with the superimposed continuum isophotes.

Table 1. Log of observations at the SAO RAS telescope

\begin{tabular}{llllll}
\hline \hline Date & Instrument/mode & Exp. time, s & Sp. range & FWHM & Seeing \\
\hline 14/15 Mar 2007 & SCORPIO/FPI & $32 \times 250$ & [O III] $] \lambda 4959$ & $1.2 \AA$ & $1^{\prime \prime} \cdot 2$ \\
22/23 Oct 2008 & SCORPIO/DI & $5 \times 120$ & $\mathrm{R}_{C}$-filter & & $1^{\prime \prime} .2$ \\
03/04 Feb 2008 & MPFS & $6 \times 1200$ & $3650-5900 \AA$ & $5 \AA$ & $1^{\prime \prime} 3$ \\
03/04 Feb 2008 & MPFS & $6 \times 1200$ & $4300-7380 \AA$ & $5 \AA$ & $1^{\prime \prime} .3$ \\
12/13 Feb 2019 & SCORPIO-2/LS & $3 x 900$ & $3650-8530 \AA$ & $7 \AA$ & $2^{\prime \prime} .7$ \\
\hline \hline
\end{tabular}

in the resulting data cube with $0.7^{\prime \prime} / \mathrm{px}$. The MPFS data cube had the $16 \times 16$ $\operatorname{arcsec}^{2}$ field-of-view centered on the nucleus with the $1^{\prime \prime} /$ spaxel spatial scale and covered a wide spectral range.

Calibration of the SCORPIO-2 and MPFS spectra into the absolute energy flux units was carried out using spectrophotometric standard stars observed in the same night as Mrk 78. The data reduction was performed in a standard way (see Smirnova et al., 2018, for references on the software and algorithms).

Fig. 2 shows the maps derived from the Voigt fitting of the [O III] spectra in the FPI data cube: the monochromatic image, the velocity field, and the velocity dispersion map free from instrumental effects. The emission-line fluxes in the MPFS and long-slit data were derived from the single-component Gaussian fitting. The map of the [O III] emission in the circumnuclear region according to the MPFS data is shown in Fig. 1. For the internal region, where well-known multi-component lines were observed (see Afanasiev \& Silchenko, 1991, and references therein), only a brighter peak was fitted in the MPFS and FPI data. 


\section{External gas clouds}

The ionization state of the EELR inside the galaxy disc $\left(r<10-15^{\prime \prime}\right)$ was analysed in the papers cited above in Sec. 1. However, the FPI map also revealed separate ionized gas clouds (labeled as $\mathrm{C} 1$ and $\mathrm{C} 2$ in Fig. 2) at a distance of $\sim 15-$ $20^{\prime \prime}(\sim 12-16 \mathrm{kpc})$ southwest of the galaxy. The line-of-sight velocity field shows that the cloud velocities lie in the range of the gas disc $\left(10900-11500 \mathrm{~km} \mathrm{~s}^{-1}\right)$. Whereas the velocities of $\mathrm{C} 1$ and $\mathrm{C} 2$ exceed by $50-200 \mathrm{~km} \mathrm{~s}^{-1}$ the nearest S$\mathrm{W}$ side of the galactic disc. This indicates that both clouds are moving in the Mrk 78 gravitational potential, but rotate out of the galaxy plane on retrograde orbits possibly.

To determine the $\mathrm{C} 1$ and $\mathrm{C} 2$ ionization state, the SCORPIO-2 long-slit spectra were obtained along $\mathrm{PA}=127^{\circ}$. The slit position has been selected according to the centers of both clouds and a neighbouring galaxy with an unknown spectroscopic redshift (see Sec. 4).

To determine the ionization mechanism of the gas in $\mathrm{C} 1$ and $\mathrm{C} 2$, the emissionline ratio diagnostic diagrams (BPT-diagrams after Baldwin et al., 1981) were used with the branches from Kewley et al. (2006) to separate different mechanisms of gas excitation: the HII-regions and AGN (Seyfert or LINER). These diagrams involve the following line flux ratios independent from the interstellar extinction: [N II] $\lambda 6583 / \mathrm{H} \alpha$, [O III $] \lambda 5007 / \mathrm{H} \beta$, and $[\mathrm{S} \mathrm{II}] \lambda 6717+6731 / \mathrm{H} \alpha$.

The integrated spectrum of the $\mathrm{C} 1+\mathrm{C} 2$ clouds is shown in Fig. 3, the BPT diagrams are presented in Fig. 4. We designated the EELR ionization mechanism according to the long-slit spectroscopy. To compare their ionization state to that of the circumnuclear region, the MPFS intergral-field spectroscopic data were used. It is clearly seen that the $\mathrm{C} 1$ and $\mathrm{C} 2$ ionization state is the same as that in the internal part of the EELR: the Seyfert-type AGN radiation predominates. This result is in agreement with the diagrams based on the long-slit spectroscopy along the major galactic axis $\left(\mathrm{PA}=90^{\circ}\right)$ presented in Keel et al. (2012).

Fischer et al. (2011) estimated the parameters of the ionized cones according to the HST data for the inner $\left(r<4^{\prime \prime}\right)$ brightest part of the emission-line structure. We overlaid the western side of this bicone (having an opening angle of $\sim 75^{\circ}$ ) on the FPI dispersion map (Fig. 2): $\mathrm{C} 1$ and $\mathrm{C} 2$ both fall into the projected cone borders. Therefore, the ionization state of the distant gas in the EELR is consistent with the model based on the analysis of the circumnuclear gas kinematics and ionization state. The ionized-gas velocity dispersion in C1 and $\mathrm{C} 2\left(40<\sigma<100 \mathrm{~km} \mathrm{~s}^{-1}\right.$, see Fig. 2) is similar to the gas in the outer part of the galaxy disc and significantly smaller than that observed in the inner region, where jet-cloud interaction takes place. It means that the external part of the EELR is dynamically cold, i.e., the AGN ionized off-plane gas clouds are related with the external accretion or tidal structures, but are not the result of the AGN outflow. The similar picture is observed in most EELRs in Seyfert galaxies (see Keel et al., 2015, and references therein). 

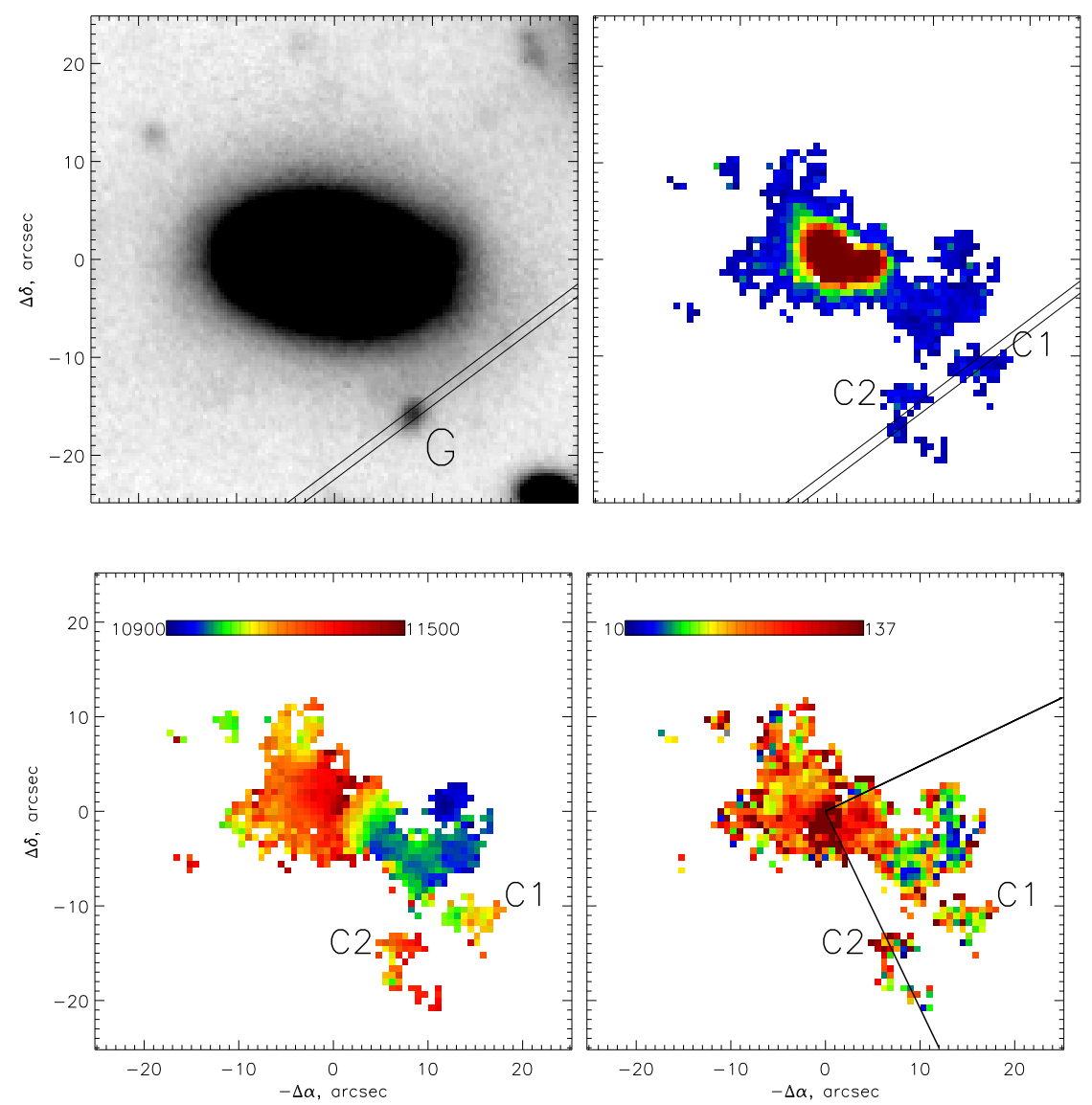

Figure 2. Mrk 78 observations. Top left: the SCORPIO $R$-band image with the long-slit position, the candidate companion galaxy is marked as ' $G$ '. Top right: the map of the [O III $] \lambda 4959$ emission derived from the FPI data. Bottom: the line-of-sight velocity field in the [O III] emission line (left) and the velocity dispersion map with the marked ionization cone position (right). The colour bars are in the $\mathrm{km} \mathrm{s}^{-1}$, the external gaseous clouds are marked as ' $\mathrm{C} 1$ ' and 'C2'.

\section{Possible dwarf companion}

Assumptions that Mrk 78 is a post-merging system were considered in a number of papers since the eighties of the last century. Morphological peculiarities on different spatial scales were considered as the galaxy interaction imprints: the nuclear dust lane (Whittle \& Wilson, 2004), the disturbed external isophotes in the S-W part of the galactic disc (De Robertis, 1987; Pedlar et al., 1989; Keel 

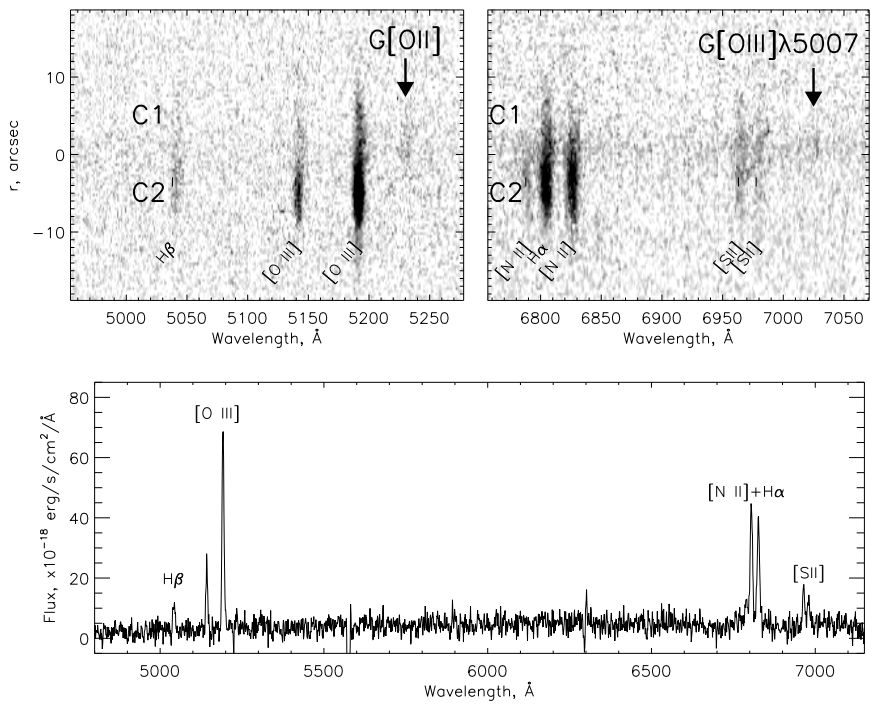

Figure 3. Top: identification of the emission lines in the ionized-gas clouds ( $\mathrm{C} 1$ and C2) and the nearby galaxy long-slit spectra. Bottom: the integrated spectrum of both clouds.

et al., 2015), the asymmetric distribution of the external [O III] emission (Unger et al., 1987; Afanasiev \& Silchenko, 1991).

De Robertis (1987) suggested that the disturbed external isophotes are caused by passing through a companion galaxy and the emission of this region due to the companion galaxy nucleus, but the forthcoming HST data disproved this idea (Fischer et al., 2011).

The SDSS DR15 and SCORPIO $R$-band images reveal the galaxy SDSS J074240.37+651021.4 with an unknown redshift in the nearest neighbourhood of the $\mathrm{C} 1$ and $\mathrm{C} 2$ clouds at a distance of $\sim 18^{\prime \prime}$ from the Mrk 78 nucleus (marked as ' $G$ ' in Fig. 2). We have checked the possibility that this galaxy is a dwarf companion of Mrk 78 or a remnant of a tidal structure. The obtained long-slit spectra (see the slit position in Fig. 2, $\mathrm{PA}=127^{\circ}$ ) show the continuum of ' $\mathrm{G}$ ' with weak emission lines $(\lambda=5230 \AA$ and $\lambda=7016 \AA)$ marked with arrows in Fig. 3. We interpreted them as $[\mathrm{O}$ II $] \lambda 3727$ and $[\mathrm{O}$ III $] \lambda 5007$ at the redshift $z=0.382$. In this case, the $\mathrm{H} \beta$ should be blended with $[\mathrm{S} \mathrm{II}] \lambda 6731$ in the $\mathrm{C} 1$ and C2 clouds. This spectroscopic redshift is comparable to the photometric SDSS DR15 (Aguado et al., 2019) redshift: $z=0.367 \pm 0.130$. Therefore, it is a distant background galaxy. 

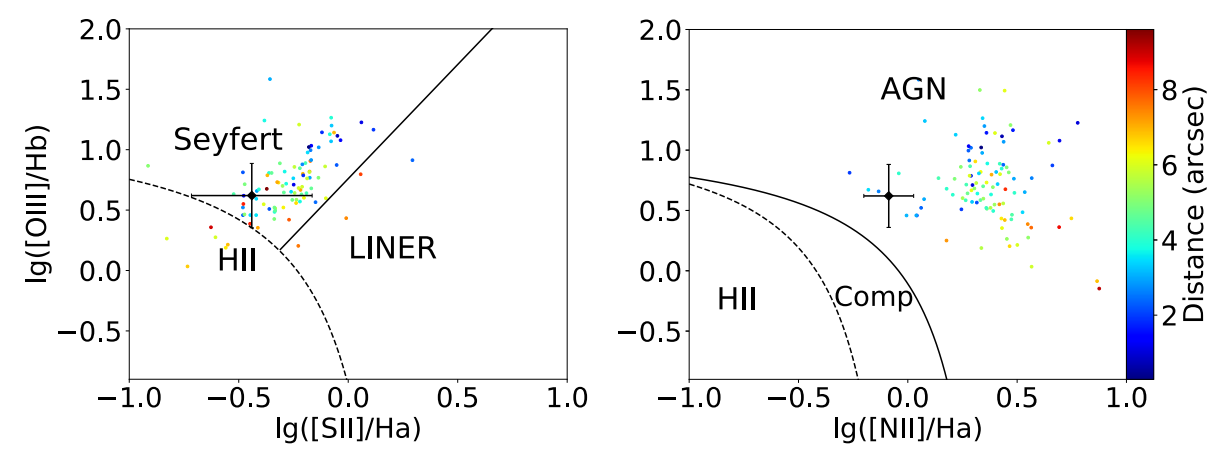

Figure 4. BPT diagrams. The separate lines are taken from Kewley et al. (2006). The circles denote the MPFS data for the circumnuclear region, the colour bar shows the projected distance of pixels on the MPFS maps from the nucleus. The diamond shows the line ratio of $\mathrm{C} 1+\mathrm{C} 2$ derived from the long-slit spectrum.

\section{Summary}

The 6-m telescope observations reveal a pair of ionized-gas clouds southwest from the stellar disc of Mrk 78 at $12-16 \mathrm{kpc}$ projected distance from the AGN located beyond the galactic disc plane. The BPT diagrams have demonstrated that the AGN radiation makes the main contribution to the ionization state of these clouds. Moreover, the location of both clouds together with other external [O III]-emitting structures in the west side of the Mrk 78 disc is in a good agreement with the ionization cone borders proposed by Fischer et al. (2011) according only to the circumnuclear region structure and kinematics.

The AGN outflow as a source of the described ionized-gas clouds is the contrary to the dynamically cold gas in this part of the EELR. The most possible scenario is an external accretion, a minor merging, or a tidal interaction with the companion. The galaxy SDSS J074240.37+651021.4 at a projected distance of $\sim 18^{\prime \prime}$ from the Mrk 78 nucleus in the southwest direction was considered a candidate. However, the long-slit spectroscopy data gave a redshift of 0.382 ; it is a distant background galaxy. Thereby, the origin of the gas in the outer part of the Mrk 78 EELRs is still unclear.

Acknowledgements. This study was supported by the Russian Science Foundation, project no. 17-12-01335 'Ionized gas in galaxy discs and beyond the optical radius' and based on the observations conducted with the 6-m telescope of the Special Astrophysical Observatory of the Russian Academy of Sciences carried out with the financial support of the Ministry of Science and Higher Education of the Russian Federation. Authors are grateful to Victor Afanasiev for his interest to this work. This research has made use of the NASA/IPAC Extragalactic Database (NED), which is operated by the Jet Propulsion Laboratory, California Institute of Technology, under contract with the National Aeronautics and Space Administration. 


\section{References}

Afanasiev, V. L., Dodonov, S. N., \& Moiseev, A. V., Kinematics of Circumnuclear Regions of Galaxies: 2D Spectroscopy on SAO RAS 6m Telescope. 2001, in Stellar Dynamics: from Classic to Modern, ed. L. P. Ossipkov \& I. I. Nikiforov, 103

Afanasiev, V. L. \& Moiseev, A. V., The SCORPIO universal focal reducer of the 6-m telescope. 2005, Astronomy Letters, 31, 194, DOI: 10.1134/1.1883351

Afanasiev, V. L. \& Moiseev, A. V., Scorpio on the $6 \mathrm{~m}$ Telescope: Current State and Perspectives for Spectroscopy of Galactic and Extragalactic Objects. 2011, Baltic Astronomy, 20, 363, DOI: 10.1515/astro-2017-0305

Afanasiev, V. L. \& Silchenko, O. K., Gas Kinematics in the Central Regions of Seyfert Galaxies - Part Six - MARKARIAN:34 MARKARIAN:78 and MARKARIAN:270. 1991, Astrofizicheskie Issledovaniia Izvestiya Spetsial'noj Astrofizicheskoj Observatorii, 33, 144

Aguado, D. S., Ahumada, R., Almeida, A., \& Anderson, S. F., The Fifteenth Data Release of the Sloan Digital Sky Surveys: First Release of MaNGA-derived Quantities, Data Visualization Tools, and Stellar Library. 2019, Astrophys. J., Suppl., 240, 23, DOI: $10.3847 / 1538-4365 /$ aaf651

Baldwin, J. A., Phillips, M. M., \& Terlevich, R., Classification parameters for the emission-line spectra of extragalactic objects. 1981, Publ. Astron. Soc. Pac., 93, 5, DOI: $10.1086 / 130766$

De Robertis, M. M., Spectroscopy and Imaging of Markarian 78 and I ZW 92. 1987, Astrophys. J., 316, 597, DOI: 10.1086/165228

Fischer, T. C., Crenshaw, D. M., Kraemer, S. B., et al., Hubble Space Telescope Observations of the Double-peaked Emission Lines in the Seyfert Galaxy Markarian 78: Mass Outflows from a Single Active Galactic Nucleus. 2011, ApJ, 727, 71, DOI: 10.1088/0004-637X/727/2/71

Keel, W. C., Bennert, V. N., Pancoast, A., et al., AGN photoionization of gas in companion galaxies as a probe of AGN radiation in time and direction. 2019, Mon. Not. R. Astron. Soc., 483, 4847, DOI: 10.1093/mnras/sty3332

Keel, W. C., Chojnowski, S. D., Bennert, V. N., et al., The Galaxy Zoo survey for giant AGN-ionized clouds: past and present black hole accretion events. 2012, Mon. Not. R. Astron. Soc., 420, 878, DOI: 10.1111/j.1365-2966.2011.20101.x

Keel, W. C., Maksym, W. P., Bennert, V. N., et al., HST Imaging of Fading AGN Candidates. I. Host-galaxy Properties and Origin of the Extended Gas. 2015, AJ, 149, 155, DOI: 10.1088/0004-6256/149/5/155

Kewley, L. J., Groves, B., Kauffmann, G., \& Heckman, T., The host galaxies and classification of active galactic nuclei. 2006, MNRAS, 372, 961, DOI: 10.1111/j.13652966.2006.10859.x

Pedlar, A., Meaburn, J., Axon, D. J., et al., Collimated radio and optical emission associated with the Seyfert galaxy Markarian 78. 1989, Mon. Not. R. Astron. Soc., 238, 863, DOI: $10.1093 /$ mnras/238.3.863 
Smirnova, A. A., Moiseev, A. V., \& Dodonov, S. N., A close look at the well-known Seyfert galaxy: extended emission filaments in Mrk 6. 2018, Mon. Not. R. Astron. Soc., 481, 4542, DOI: 10.1093/mnras/sty2569

Unger, S. W., Pedlar, A., Axon, D. J., et al., The extended narrow-line region in radio Seyferts : evidence for a collimated nuclear UV field ? 1987, Mon. Not. R. Astron. Soc., 228, 671, DOI: 10.1093/mnras/228.3.671

Whittle, M. \& Wilson, A. S., Jet-Gas Interactions in Markarian 78. I. Morphology and Kinematics. 2004, $A J$, 127, 606, DOI: 10.1086/380940 